\title{
Health Monitoring of Composite Structures via MEMS Sensor Networks: Numerical and Experimental Results ${ }^{\dagger}$
}

\author{
Stefano Mariani ${ }^{1, *}$, Giovanni Capellari ${ }^{1}$, Francesco Caimmi ${ }^{2}$ and Matteo Bruggi ${ }^{1}$ \\ 1 Department of Civil and Environmental Engineering, Politecnico di Milano, Piazza L. da Vinci 32, \\ 20133 Milano, Italy; giovanni.capellari@polimi.it (G.C.); matteo.bruggi@polimi.it (M.B.) \\ 2 Department of Chemistry, Materials and Chemical Engineering “Giulio Natta”, Politecnico di Milano, \\ Piazza L. da Vinci 32, 20133 Milano, Italy; francesco.caimmi@polimi.it \\ * Correspondence: stefano.mariani@polimi.it \\ + Presented at the 5th International Symposium on Sensor Science (I3S 2017), Barcelona, Spain, \\ 27-29 September 2017.
}

Published: 4 December 2017

Laminated composites often develop hidden damages, e.g., delamination. Such events can be effectively sensed through embedded structural health monitoring (SHM) systems, taking advantage of the interlaminar regions to place sensors; experimental campaigns proved that this approach may turn out to increase the sensitivity to small defects and reduce the remaining lifetime of the structure. In former studies, we proposed the adoption of a surface-mounted SHM system based on (inertial) MEMS sensors, which has the advantages of low cost and of suppressing the mentioned effects on lightweight structures. On the other hand, the relatively low accuracy of MEMS sensors may hinder reliable monitoring of the system state; this can be overcome through redundancy and an efficient sensor placement. An automatic approach is presented to define the optimal topology of a network featuring a limited number of sensors, wherein the extent and location of stiffness degradation due to damage are assumed to be unknown. The goal of the optimization procedure is to maximize the overall sensitivity to damage of the measurements collected through the whole SHM system. The method has been implemented in a multi-scale frame, to efficiently handle sensors, damaged regions and structural components of different sizes. Although based on deterministic modeling, results are provided to show how measurement noise can be dealt with; a comparison with a stochastic approach based on Bayesian experimental design is provided too. Experimental data collected by testing composite specimens and panels are finally discussed, to assess the identifiability of damage through the collected (noisy) measurements.

Conflicts of Interest: The authors declare no conflict of interest.

(C) 2017 by the authors. Licensee MDPI, Basel, Switzerland. This article is an open access article distributed under the terms and conditions of the Creative Commons Attribution (CC BY) license (http://creativecommons.org/licenses/by/4.0/). 